\title{
What can we see from Investment Simulation based on Generalized $(\mathrm{m}, 2)$-Zipf law?
}

\author{
Hokky Situngkir \\ (hs@compsoc.bandungfe.net) \\ Dept. Computational Sociology \\ Bandung Fe Institute \\ Yohanes Surya \\ (yohaness@centrin.net.id) \\ Surya Research International
}

(April 27, 2005)

\begin{abstract}
The paper revisits the investment simulation based on strategies exhibited by Generalized $(m, 2)$-Zipf law to present an interesting characterization of the wildness in financial time series. The investigations of dominant strategies on each specific time series shows that longer words dominant in larger time scale exhibit shorter dominant ones in smaller time scale and vice versa. Moreover, denoting the term wildness based on persistence over short term trend and memory represented by particular length of words, we can see how wild historical fluctuations over time series data coped with the Zipf strategies.
\end{abstract}

Keywords: Generalized (m,2)-Zipf law, time series, fluctuations, investment. 


\section{Introduction}

In order to choose financial products for investment (e.g.: stock indexes, future exchange indexes, foreign exchange indexes) one often aware of how fast a particular financial values change over time by referring to its historical time series. There are commonsensical thoughts that some markets are very "wild" for price varies so fast and the other is not of price moves very slowly. From the bottom-up perspective, it is apparent that the price movement will eventually remain the market depth liquidity, i.e.: order flow necessary to move price by a given amount over time. In return, other thing that also contributed to this issue is the 'market climate' surrounding investors or traders triggering the herding behavior over the market [6].

Ausloos and Bronlet [1] previously have described the way of texifying the time series data and perform statistical aspects revealing the Zipf law. In advance, they also propose an interesting investment strategy based on their findings on power-laws inspired by the so-called DFA method. The Zipf law of the financial time series is presented by transforming the ups and downs of financial index fluctuations into "words" of $m$-length and $k$-types of "letters" [4]. Obviously, there are a lot of possibilities on representing the financial indexes into texts, but the rest of the paper will use the letter " $u$ " for higher value over time respect to the price in the opening and closing session of the market and " $d$ " for downward price movement - whether they can be weekly, daily, sessional (morning and afternoon market sessions), and even smaller time intervals, e.g.: hour, minutes, seconds [8]. However, concerning the investment simulation, we will use the smallest interval of hourly [7].

The paper wants to open a new possible analytical door on financial market by bridging the investment-based paradigm and the adherence of the generalized Zipf law. We see a possibility on a new and important way to approach, characterize and extract information within the time series data by analysis on the texified fluctuations. The next section of the paper elaborates the Zipf law in financial data in the way we have the simulated investment results by using the Generalized Zipf law. This is followed by discussions on "wildness" of the time series data concerning the appropriate value of $k$ used in the simulation. 


\section{Text from Time Series}

By representing the price fluctuations into, say two alphabets, we have possible words in the time series data equal to $2^{m}=2^{2}$ possible words. They are "uu", "dd", "ud", and "du". This is applied up to several numbers of possible words. Thus, we sort frequency of occurrence of the words along our data to have the illustration of the Zipf plot - rank the sequence started from $R=1$ for the most frequent words. According to Zipf law, we will have relation of:

$$
f \simeq R^{-a}
$$

In accordance with R/S analysis, Bronlet, et. al. [4] showed the conjecture relation:

$$
a=|2 H-1|
$$

It is important to note that the probability of ups (u) and downs (d) of price in most of the case is not the same for every time series data. Here, we found bias:

$$
\varepsilon=p(u)-p(d)
$$

where $p(n)$ and $p(t)$ each is the probability of ups and downs in time series data. In this case, we need to put into account the frequency of expectation $f^{\prime}$, which represent random situation (un-correlated). In this case, in our calculation we use $f$ ' as the substitution from the original form of Zipf $(f)$ method with coefficient of exponent $a^{\prime}$. This mathematically can clearly prevent the presence of exponent $a=0$ which is possible to occur since those biases. The value of $f^{\prime}$ can be calculated as

$$
f^{\prime}=p^{m-n}(u) \cdot p^{n}(d)
$$

By now, we can start investment simulation for some financial data from various markets. This is aimed for us to have basic of prediction based on scaling character from each financial data in case of investment. Technically, the question is: what would likely to happen if we have words sequence with alphabet sequence length of $(m-1)$ ?

If we have words sequence of $c(t-m+1), \ldots, c(t-1), c(t)$, how big the probability of $c(t)$ to be "up" or "down", with $c(t)$ is showing the characters of time $t$. From this point, we calculate the prediction based on 
the words we have ranked in Zipf plot to find the level of probability of up, $p_{u p}(t)$, that is the occurrence of sequence $c(t-m+1), . ., c(t-1)$, " $u$ ", and the probability of down, $p_{\text {down }}(t)$, or the occurrence of the sequence $c(t-m+1), . ., c(t-1), " d "$. It is suggested [7] to consider variable of strength parameter that is how far we can trust a calculation result, which is through relative probability represented by:

$$
D(t)=\left|\frac{p_{\text {up }}(t)-p_{\text {down }}(t)}{p_{\text {up }}(t)+p_{\text {down }}(t)}\right|
$$

This $D(t)$ parameter shows how big the probability of the prediction result to have value within interval [0..1]. In the simulation, we use parameter $D(t)$ as a form of fraction of how many we "buy" or "sell" the index value. As we know, we will pose to "buy" when $p_{u p}(t)>p_{\text {down }}(t)$ and vice versa. By the simulation, simply we can write the outcome of investment after $\mathrm{n}$ time as

$$
\psi_{\text {total }}=\psi_{\text {start }}+\sum_{i=2}^{n}\left(p_{i}-p_{i-1}\right) D_{i}
$$

while in each time-steps we have

$$
\psi(t)=\psi(t-1)+[p(t)-p(t-1)] D(t)
$$

An example of our simulation result is depicted in figure 2 preceding the Zipf law in Jakarta Composite Index (IHSG), DJIA, and NASDAQ on each daily data in figure 1. Obviously, the figure shows that the dominant investment strategy in IHSG is Zipf $(6,2)$ while in DJIA and NASDAQ are Zipf (4,2) and Zipf (5,2) respectively.

\section{On wildness of financial time series data}

After transforming a series of financial data into text and having simulation result based on Zipf law in hand, plenty of analytical thoughts may appear. The notion of relative wildness of financial data can be treated as the way we cope with the possibilities of ups and downs of the existing fluctuations. Obviously, when a time series data can be 'read' well by the Generalized Zipf law, we have a good view to cope with the wildness of the time series. Furthermore, the length of the words suitable to gain better along the certain time window of investment enriches our notion of this relative 'wildness'. 
Here we can see how persistent a word along certain time. For example, if a particular time series data get better gain while we use the rule of investment of Zipf (7,2) relative to the one of Zipf (2,2), then we can say that the wildness of the one with Zipf (7,2) is wilder than the other one. In other words, we can say that concerning the short term trend persistence, time series represented by 7 words in investment is less wild relative to the one with 2 words.

In advance, we can also assume that the persistence over particular words as a memory effect of time series data. In this perspective, apparently a less wild time series has longer memory since the persistent trend is longer. This is very interesting when we try to see the effect in several specific financial data, e.g.: financial indexes and foreign exchange rates.

In figure 3 and 4 , we show the result of our investigation in daily Yen/USD and Euro/USD data showing good gain in Zipf (3,2) and Zipf (2,2) respectively. Comparing to the previous one in figure (2), we can say relatively that both foreign exchange rates exhibit wilder fluctuations regarding persistence over trends and memory represented by the specific words. Certainly, we can understand this since foreign exchange involves more traders with higher sensitivity to fundamental issue relative to the market indexes.

The later question is whether or not the better strategies in a certain financial products are persistent over different time scales. We do the similar analysis to HangSeng Index, NIKKEI225 index, and GPB/USD rate over different time orders. Figure (9) and (12) shows our results with HangSeng and NIKKEI225 indexes on respective time series data depicted in figure (7) and (10). Interestingly, a wilder time series with shorter dominant words in small time scale have longer words of dominant strategies in the larger time scale and vice versa. This is showed by HangSeng Index whose dominant strategy of Zipf (7,2) in daily data shrinks upto Zipf (4,2) in sessional data and Zipf $(3,2)$ in hourly in our investment simulation. In return, the dominant Zipf $(3,2)$ in daily NIKKEI225 investment simulation exhibits longer Zipf (6,2) in sessional and hourly trading. This is very interesting while GBP/USD rate shows similar situations of $\operatorname{Zipf}($ 7,2) in daily data and Zipf (8,2) in hourly simulations.

Intuitively, by referring to the wildness of a time series, we could say that there could be a pattern presented here. The time series shows less 'wildness' in a certain time scale could have wilder in bigger or narrower time scale. This opens further investigations in spite of the fact that the Generalized Zipf analysis could yield a good prediction of time series fluctuations. 


\section{Concluding Remarks and Further Works}

We show the simulation result of investment by using the strategies laid upon our understanding of Zipf law in texified financial fluctuation. We evaluate the different strategies regarding the length of words used in each simulation and discover interesting properties over different time series data and different time scale. In our simulations we can see that longer words dominant in larger time scale exhibit shorter dominant ones in smaller time scale and vice versa. In the other hand, we present that some financial indexes are wilder than other respect to the persistence of short term trend (represented by the words or sequence of letters) and memory over fluctuation patterns.

We expect to have more properties on doing several further investigations by involving more letter $(k>2)$ and by trying to relate the findings with terminologies often used to analyze the memory, distributions, and correlations of the time series. Here, notwithstanding, we have seen some possible and interesting findings of the textual analysis upon texified time series data more than the advantage of the analysis as a good and promising prediction method.

\section{Acknowledgement}

Authors thank M. Ausloos for some important literatures, Yohanis and Jackson Silaban for financial data, Tiktik Dewi Sartika for some typeset corrections, and BFI colleagues for discussions. Noone but authors are responsible for possible errors and omissions.

\section{References}

[1] Ausloos, M., and Bronlet, Ph. (2002). "Strategy for Investment from Zipf Law(s)". Physica A 324:30-7.

[2] Ausloos, M., and Ivanova, K. (1999). "Precise (m,k)-Zipf diagram analysis of mathematical and financial time series when $\mathrm{m}=6, \mathrm{k}=2$ ". Physica A 270:526-542

[3] Baxter, M., and Andrew, R. (1997). Financial Calculus: An Introduction to Derivative Pricing. Cambridge UP. 
[4] Bronlet, Ph., and Ausloos, M. (2004). Generalized $(m, k)$-Zipf Law for Fractional Brownian motion-like Time Series with or without Effect of an additional linear trend. Pre-print: arxiv:cond-mat/0209306

[5] Gammel, B. M. (1998). "Hurst's rescaled range statistical analysis for pseudorandom number generators used in physical simulations". Physical Review E 58(2):2586-97.

[6] Situngkir, H., and Surya, Y., (2004). Agent-Based Model Construction in Financial Economic System. Working Paper WPA2004. Bandung Fe Institute. Pre-print: arxiv:nlin.AO/0403041.

[7] Situngkir, H., and Surya, Y. (2005), Simulasi Investasi dengan Hukum Pangkat Zipf: Analisis Zipf- $(m, 2)$ dalam Teks Data Indeks Keuangan. Working Paper WPC2005. Bandung Fe Institute.

[8] Vanderwalle, N., Brisbois, F., and Lefebvre, P.H. (2000). "Managing Both Sign and Size of Fluctuations within the n-Zipf Framework". International Journal of Theoretical and Applied Finance 3(3):409-414. 

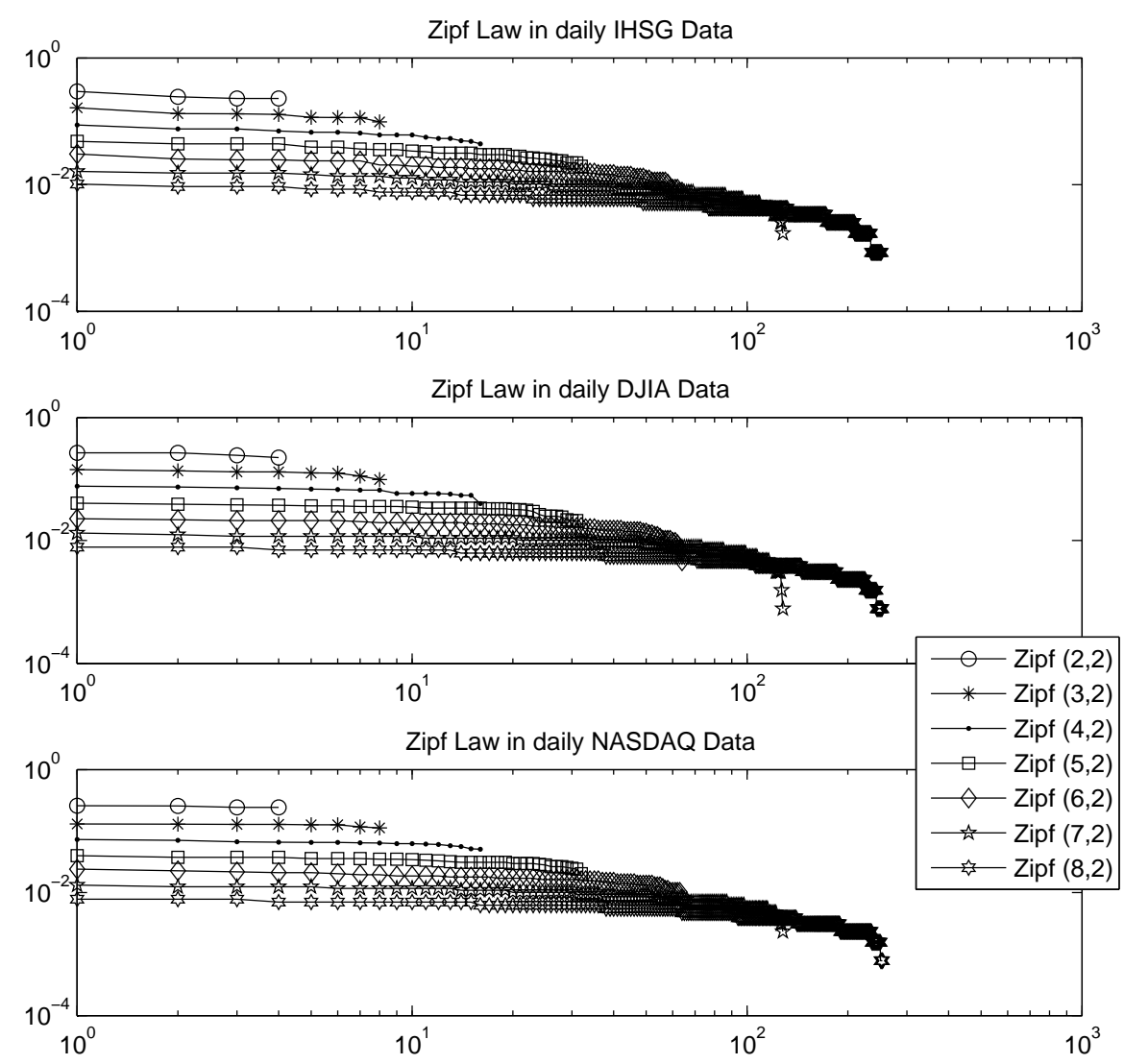

Figure 1: Zipf law for various length of words in Jakarta Composite Index (IHSG), DJIA, and NASDAQ. The data is in interval: IHSG (January 4, 2000 - November 2, 2004), DJIA (January 3, 2000 - February 14, 2005), and NASDAQ (January 3, 2000 - February 14, 2005) 

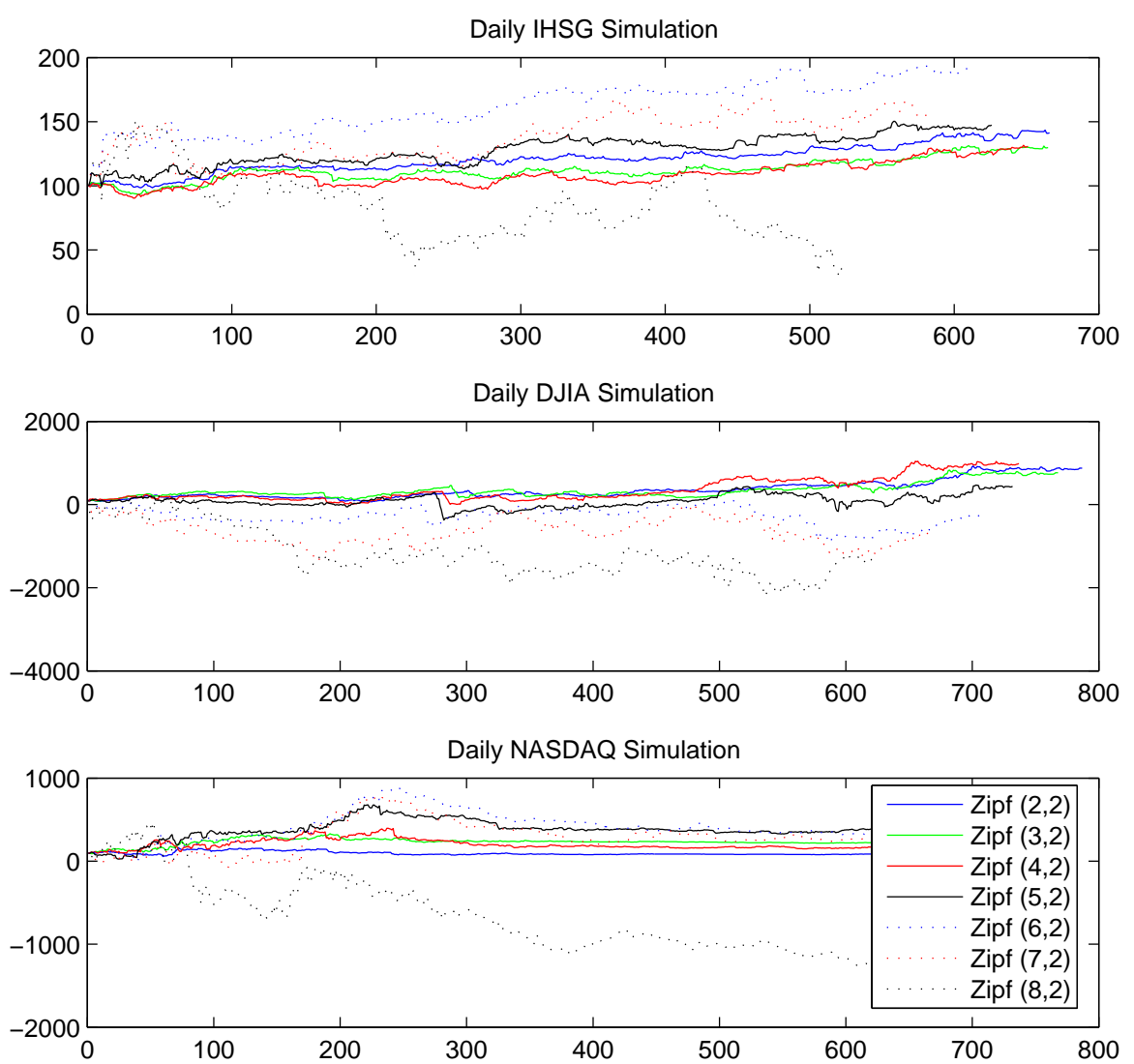

Figure 2: Investment Simulation Result showing the gained point by using the series of Jakarta Composite Index (IHSG), DJIA, and NASDAQ 

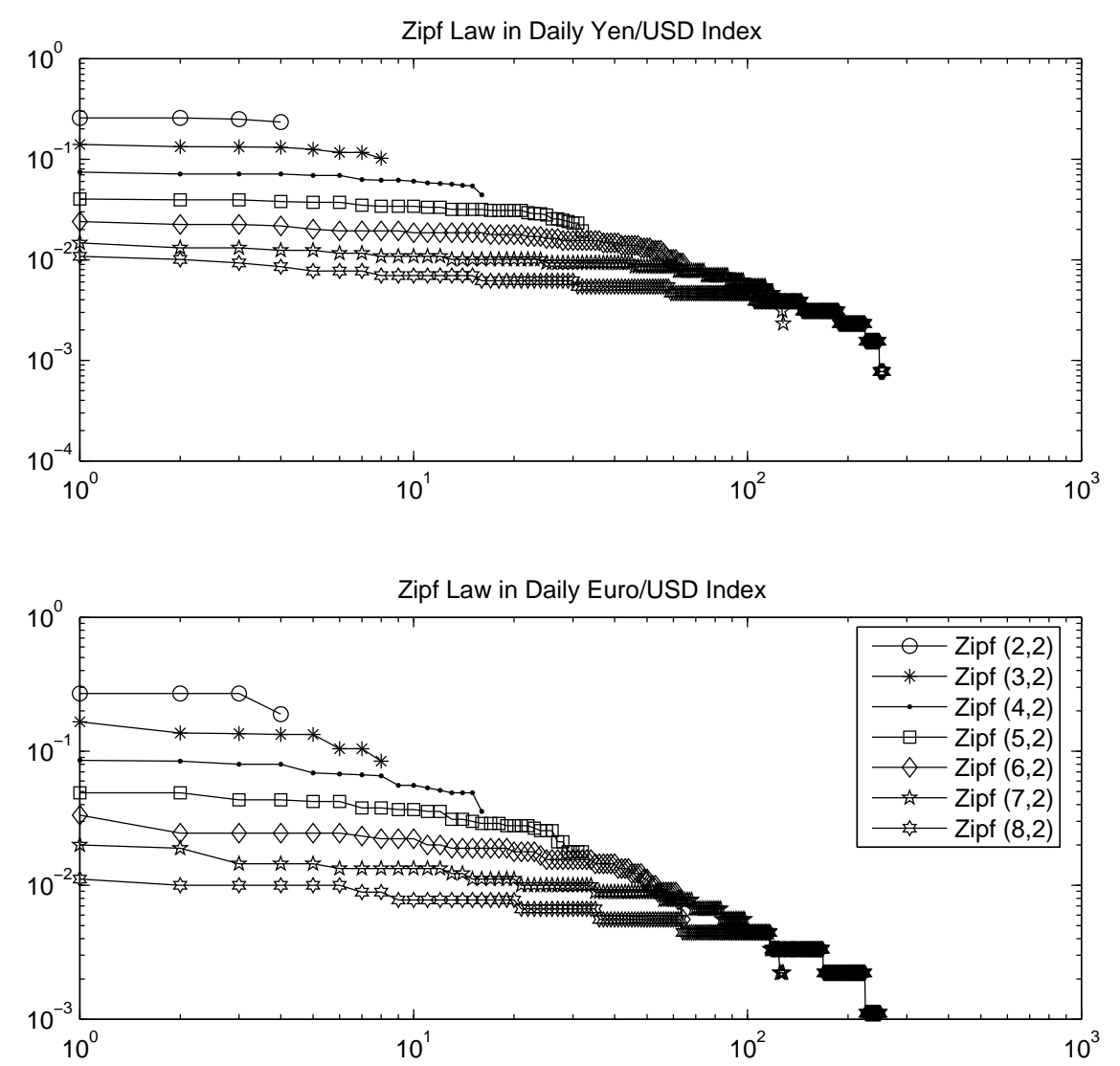

Figure 3: Zipf Law in Yen/USD rate and Euro/USD Rate. The data is in interval: Yen/USD (January 3, 2000 - December 14, 2004) and Euro/USD (July 9, 2001 - December 17 2004) 

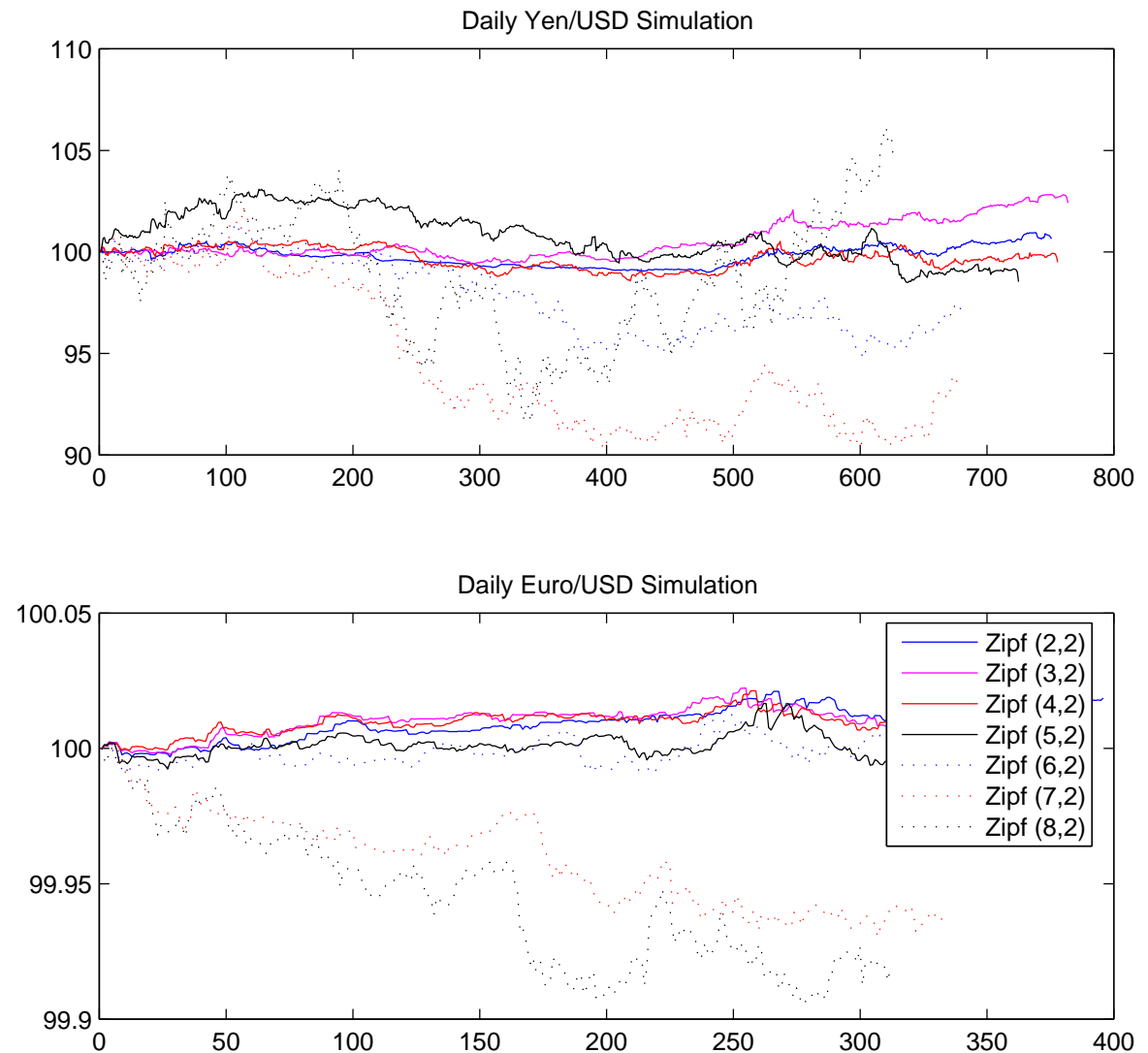

Figure 4: Investment Simulation Result showing the gained point by using the series of Yen/USD and Euro/USD rate. 

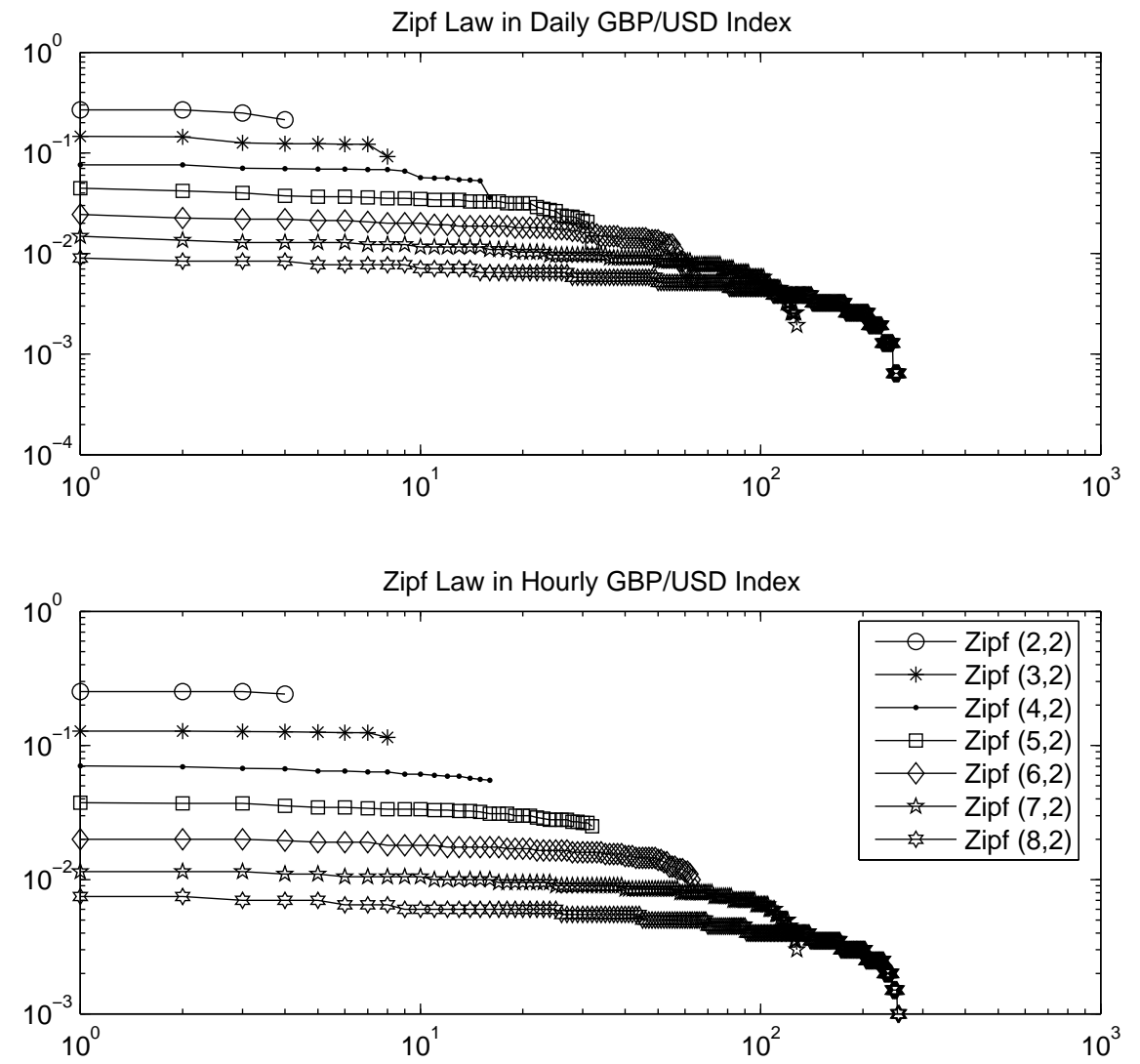

Figure 5: Zipf Law in GBP/USD Rate for daily (January, 3, 2000 - December 14 2004) and hourly data (22:00 September 30, 2004 - 12:00 January 242005. 

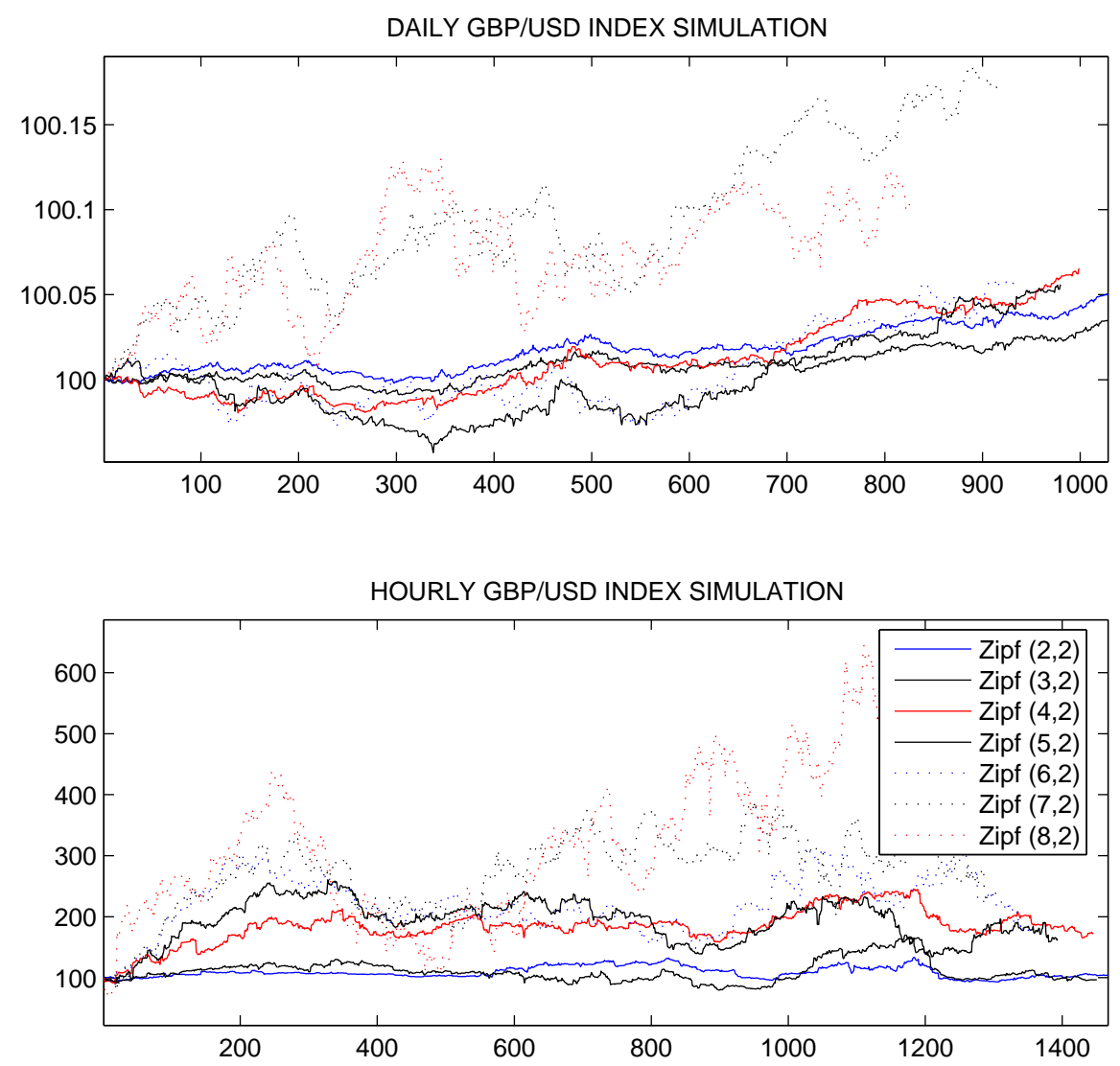

Figure 6: Investment Simulation Result for daily and hourly trading. 

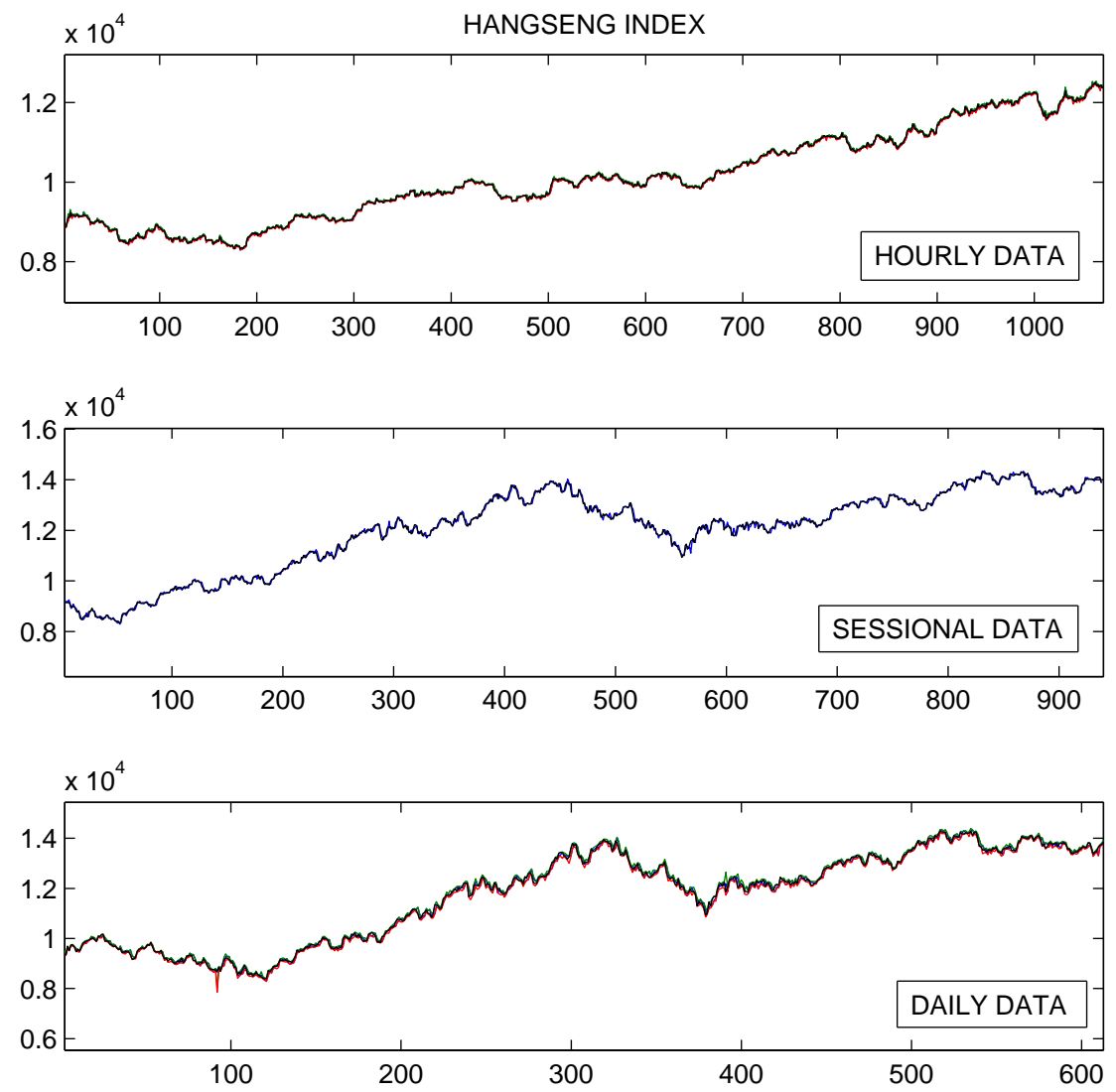

Figure 7: HangSeng Index used in investment simulation for daily (October 30, 2002 - April 26, 2005), sessional (March 19, 2003 - September 3, 2004), and hourly (10:00 March 19, 2003 - 16:00 August 28, 2003) data respectively. 

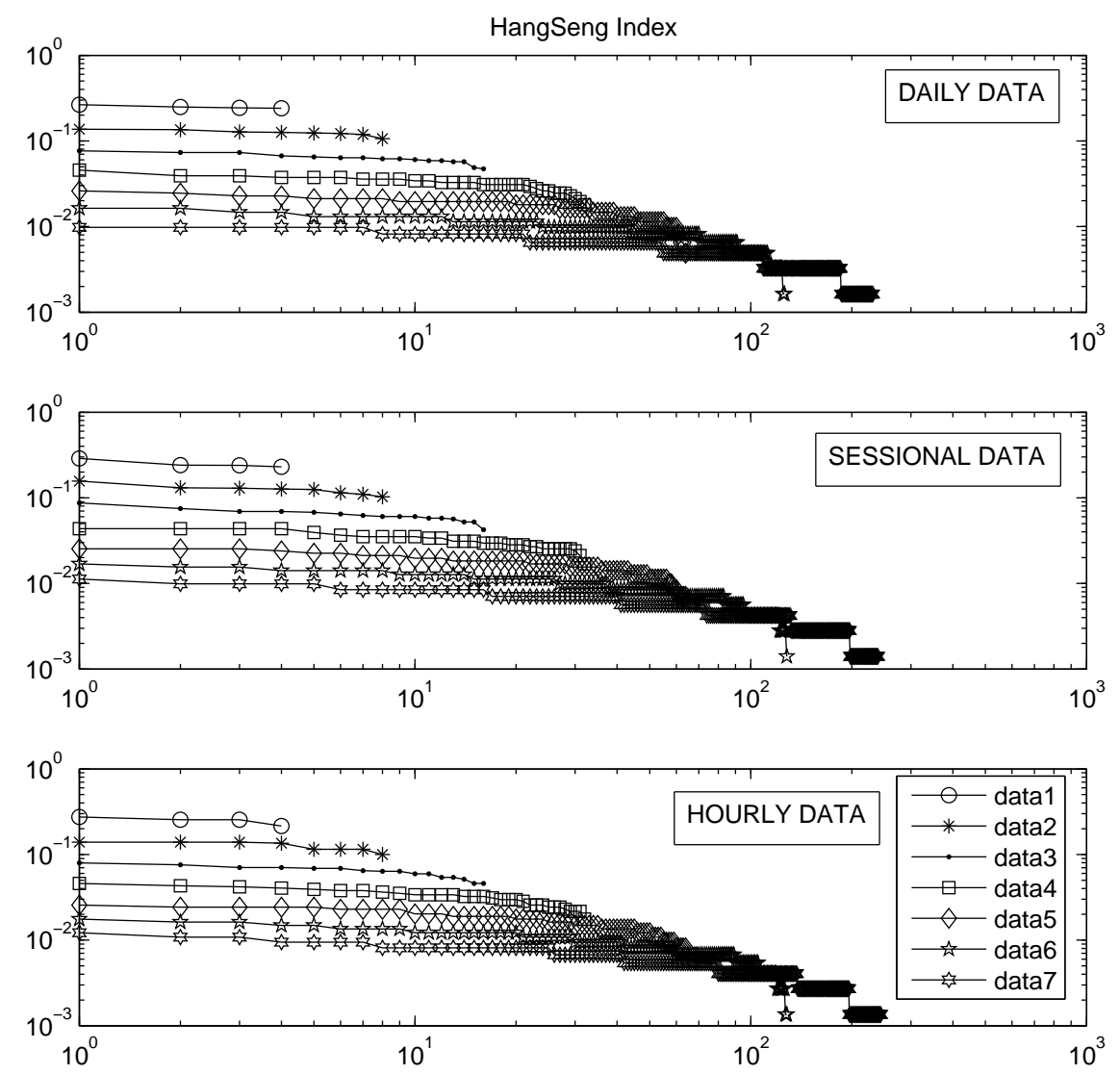

Figure 8: Zipf Law in texified HangSeng Index. 

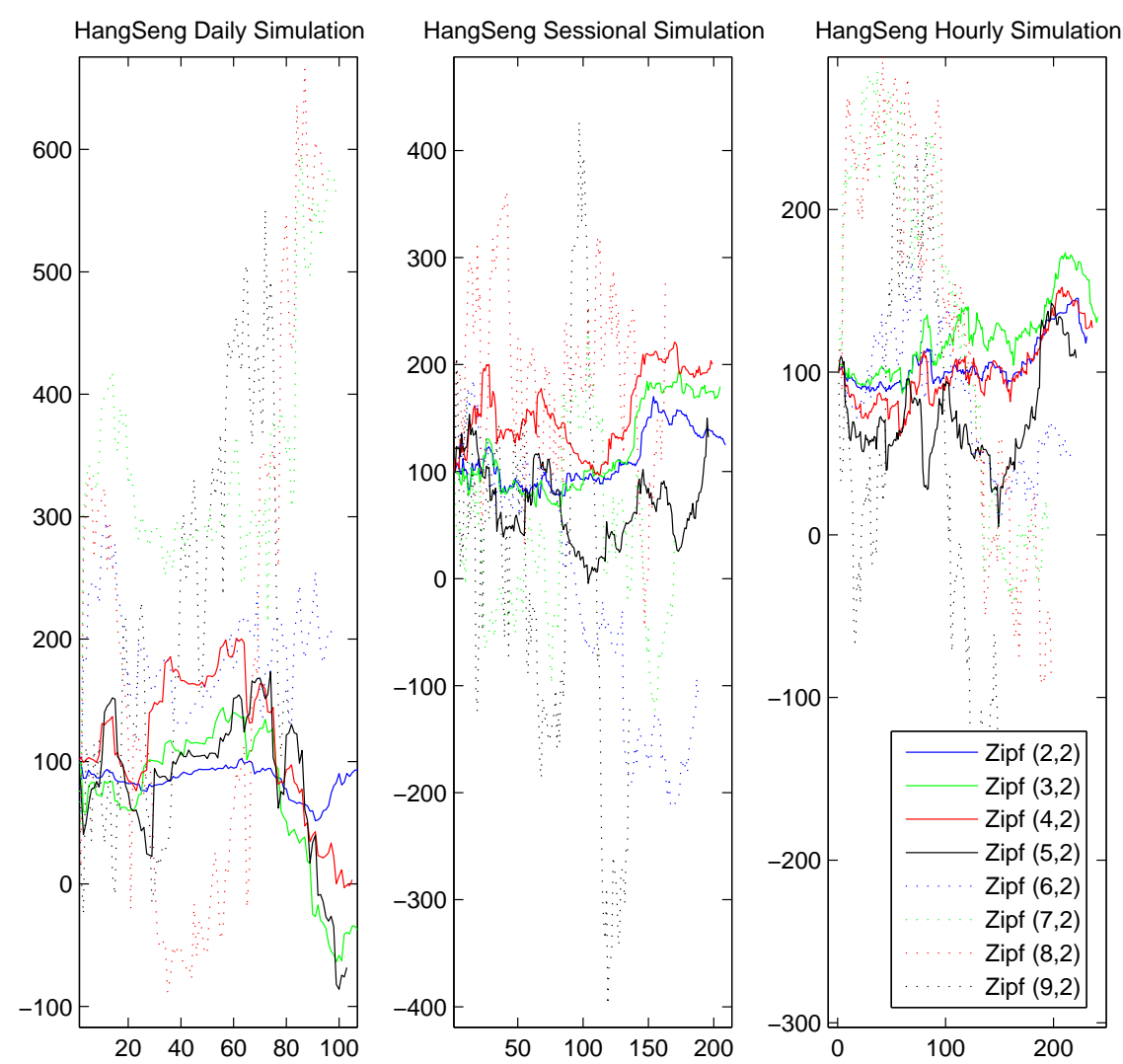

Figure 9: Simulation result comparing investment by respective $(\mathrm{m}, 2)$-Zipf law in daily, sessional, and hourly HangSeng market. 

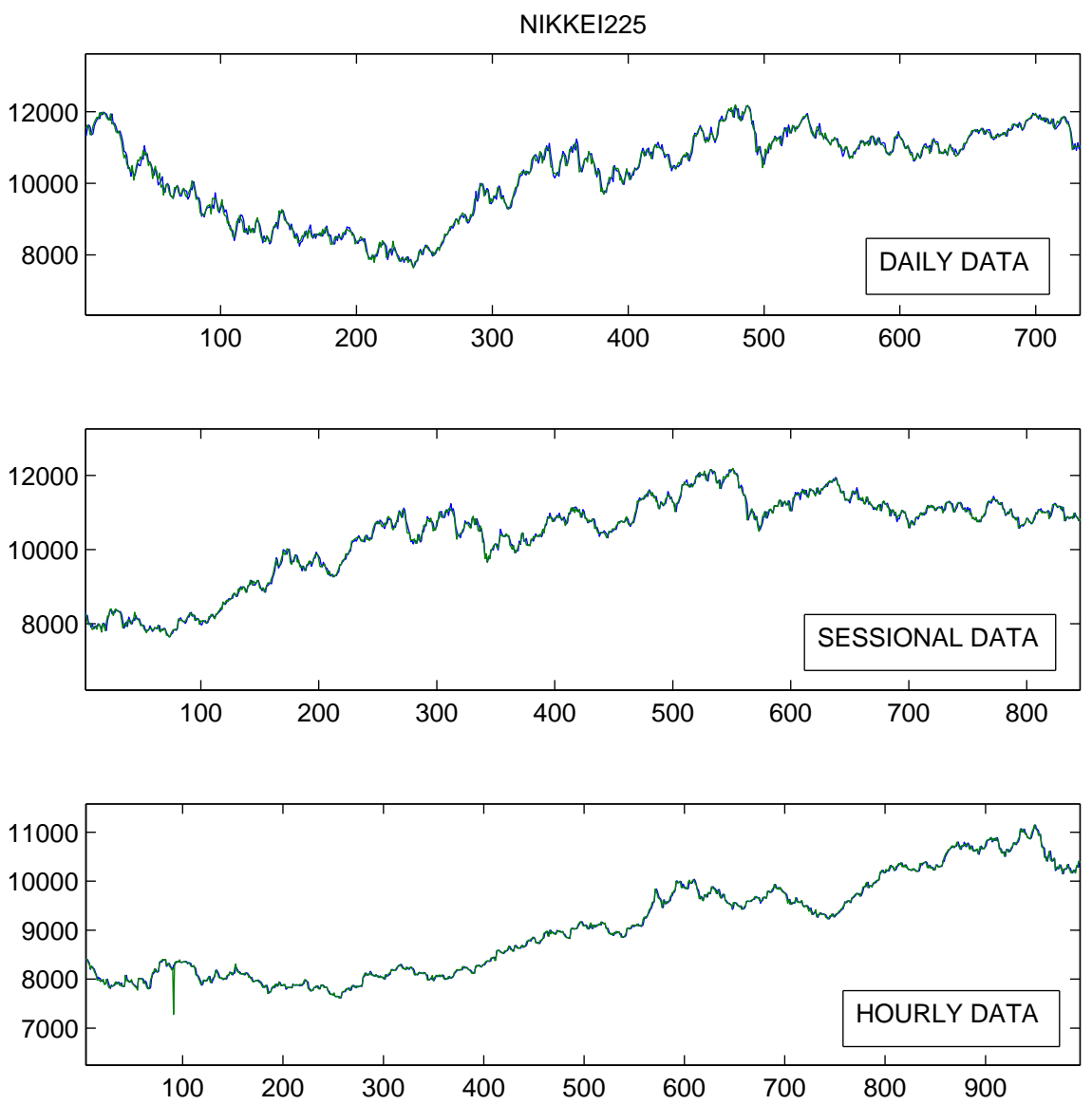

Figure 10: NIKKEI225 Index used in investment simulation for daily (May 8, 2002 - April 26, 2005), sessional (March 6, 2003 - December 2, 2004), and hourly (8:00 March 6, 2003 - 12:00 October 1, 2003) data respectively. 

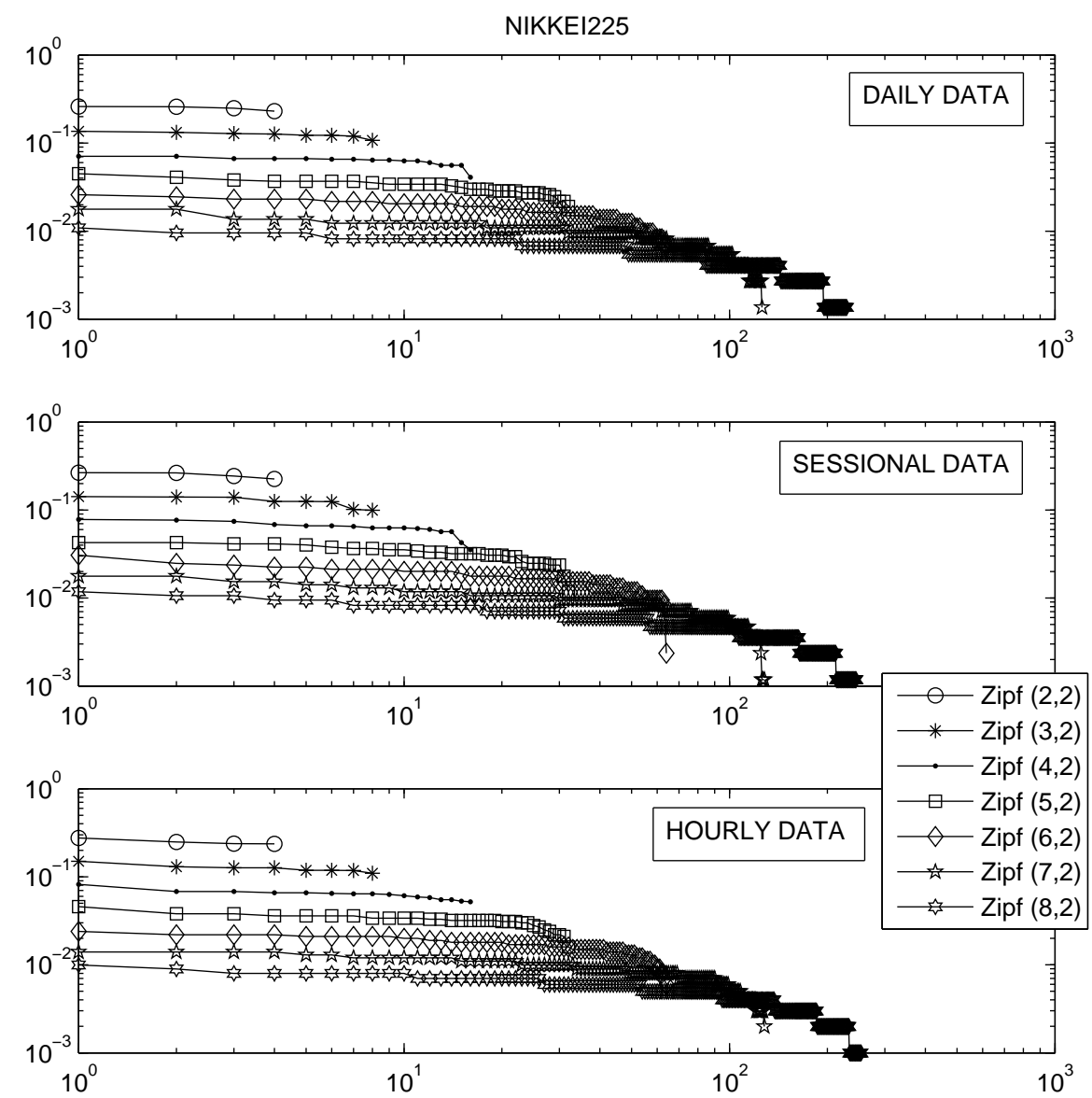

Figure 11: Zipf Law in texified NIKKEI225 Index. 


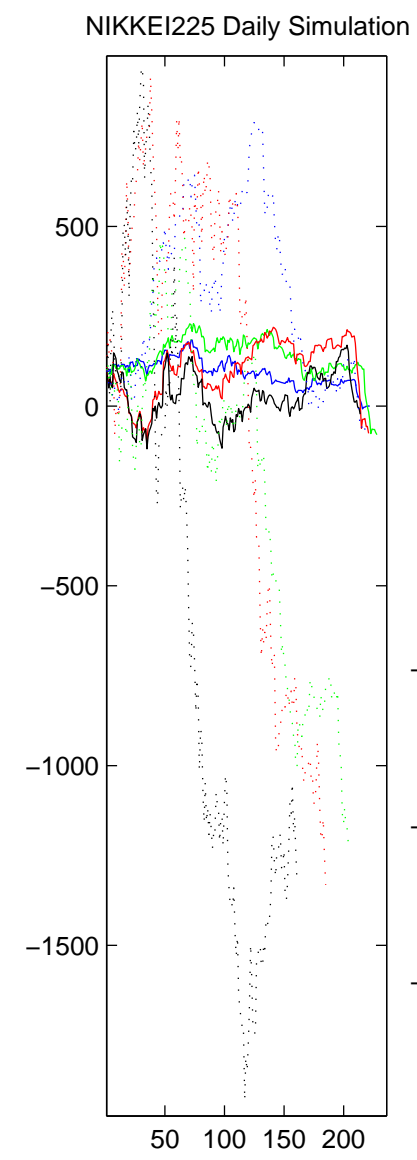

NIKKEI225 Sessional Simulation NIKKEI225 Hourly Simulation
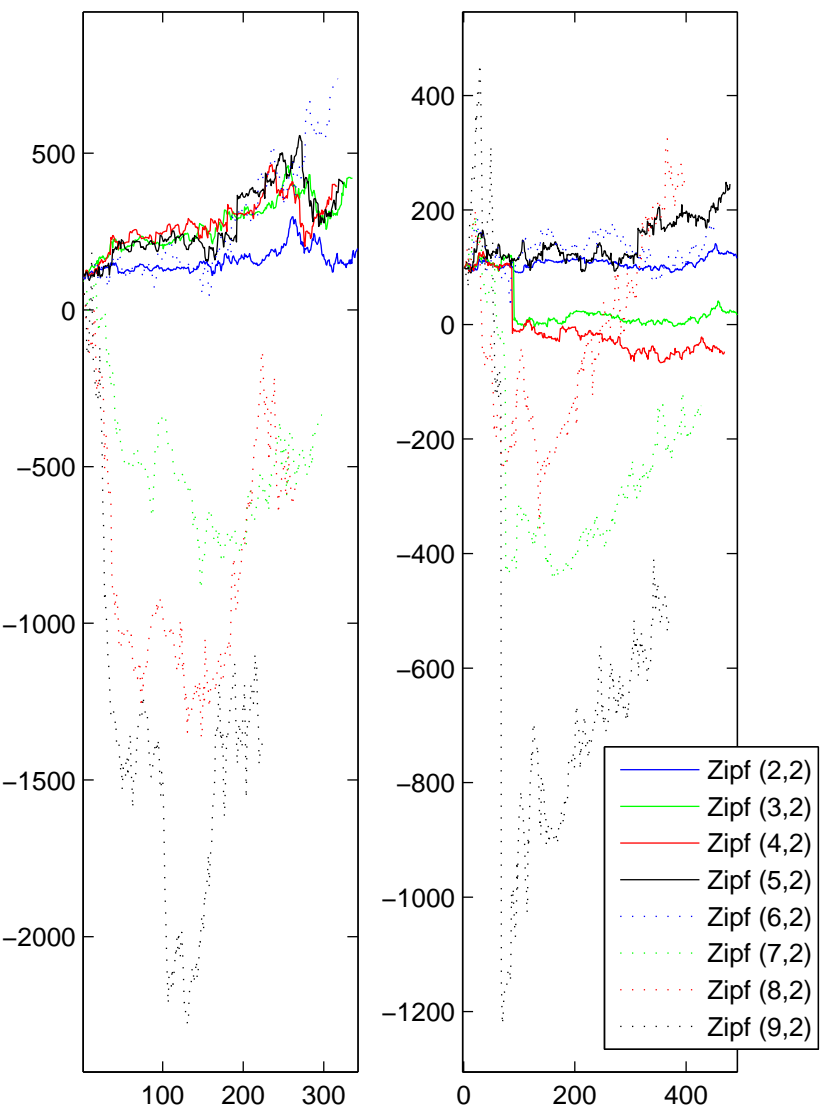

Figure 12: Simulation result comparing investment by respective $(\mathrm{m}, 2)$-Zipf law in daily, sessional, and hourly NIKKEI225 market. 